\title{
Industrial Application of Thixomet Image Analyzer for Quantitative Description of Steel and Alloys Microstructure
}

\author{
Alexander A. Kazakov ${ }^{1}$, Daniil Kiselev ${ }^{1}$ \\ ${ }^{1 .}$ St.Petersburg State Polytechnical University/Metallurgical Technologies Department, \\ St.Petersburg, Russian Federation
}

There are numerous examples of practical use in industry of the Thixomet image analyzer for quantitative description of the microstructure of steels and alloys for various purposes.

In 1996, the very first Thixomet image analyzer unit was installed at the Alumax technical center (Golden, CO) for the quantification of the structure of semi-solid materials [1]. Along with the evaluation of the distribution of porosity, eutectic content (continuous and occluded) and silicon content across the billet, a special grain shape factor was calculated in studies of anodized specimens examined in polarized light. The identification and integration of the dendrite fragments, revealed in a planar image, into one structurally isolated object was possible as a result of this measurement. Thixomet software performs a total quantitative description of 2D SSM structure, as well as direct visualization and measurement of $3 \mathrm{D}$ structure by processing data obtained using serial sections.

Since 1997 the Thixomet has been used by many Russian turbine blade plants for quality estimation of the nickel-based superalloy charge billets [2]. The total impurity rate is calculated with regard to the contribution of each impurity type: oxide films, slag globules, nitride clusters. Their recognition is based on the grayscale level of the impurity, their morphology and the character of mutual arrangement. The rate for nitride clusters is 10 times higher than for slag globules and oxide films in the same dimensional group since nitrides and their clusters are more difficult to remove from melts than slag globules or oxide films, which are easily assimilated by the refractory lining of the crucible during remelting or by ceramic filter during pouring. The decision to use the charge billet in the production of turbine blades is based upon the metallurgical quality evaluation results. Billets with an impurity rate above a defined critical value are withdrawn from production.

A motorized hardware-software complex, the "Thixomet SmartDrive", was developed and installed in dozens of enterprises and companies to provide an objective quantitative estimation of all types of structural inhomogeneity in modern pipeline steels, such as microstructural banding, general anisotropy, blocks of bainite with lath morphology and centerline segregation [3-5].

Microstructural banding is determined according to GOST 5640-68, a chart method based upon the principle of increasing the number of bands of the second phase, taking into account their continuity and degree of ferrite grain elongation. The assigned grade is based on stereological parameters that have been created using a directed secant method with the assistance of the automatic image analyzer. It should be noted that comparisons between standard chart images and those of the specimen were conducted at $\times 100$, according to GOST 5640-68, while analysis of elongated ferrite grains required $\times 500$. Such an analysis is possible only by sequential examination of the specimen at two magnifications. In classic metallography, it is impossible to observe the same wide field of view provided by $\times 100$ at $\times 500$. Therefore, classic metallographic investigations are always a compromise between the examination area and the resolution. But, if we apply modern methods of quantitative metallography using the Thixomet ${ }^{\circledR}$ image analyzer, we can simultaneously evaluate both banding and ferrite grains elongation making measurements only at $\times 500$, while the required area corresponding to the size of the field viewed at $\times 100$ can be subsequently "stitched" together electronically from adjacent fields of view. When a motorized microscope stage moves to the next 
field of view, the previous field is stitched precisely, "pixel to pixel" to the field that was captured just before. This way a high resolution "panoramic image" of any desired large area can be created. The standard deviation of the fraction of a second phase on secants that are parallel to the rolling direction devided by the standard deviation of the fraction of second phase on secants that are perpendicular to the rolling direction will unambiguously characterize structural banding without depending on the morphology of particles of the second phase.

Modern pipeline steels are manufactured using thermomechanical treatment technology which includes accelerated cooling after hot rolling producing plate with an almost $100 \%$ bainitic structure free from microstructural banding. However, anisotropy of the bainitic structure can be observed in such steels. The stereological methods that have been described above for the evaluation of ferritepearlite and ferrite-bainite microstructure are not applicable for the description of complicated bainitic morphologies. Hence, a method for evaluation of bainite anisotropy based on texture analysis of images has been developed for this case. Using the methods of texture analysis, we have no need to select any constituent of microstructure; therefore, we can evaluate microstructure with a complicated morphology and increase evaluation objectivity. The ratio determined via this method characterizes unambiguously the general anisotropy of the microstructure of bainitic steels.

The results of the investigations revealed the blocks of bainite with lath morphology among different morphological forms elongated along the rolling direction. These bainitic blocks with a lath morphology are the main contributor to general anisotropy and decrease the essential mechanical properties of the plate in the transverse direction. The technique of color etching with consequent analysis in polarized light has been developed and covered by a Russian Federation patent; it promotes perfect selection of the bainite blocks with the lath morphology and facilitates measurement of their volumetric content and the length of longitudinal inter-phase boundaries which mainly determine the mechanical properties of high strength pipeline steels.

The parameters of centerline segregation are decisive in the formation of the mechanical and corrosion-resistant metal properties. The GB/T 13298 technique assigns classes for hot-rolled plate structure based upon research results of its central area at a magnification of $\times 200$. Additionally, the structure is evaluated at $\times 500$ magnification to assess the non-metallic inclusions that are decorating the band. Such inclusions or a wide single band can be the basis for an assignment of an additional 0.5 class penalty. Class 1 defines a structure with slightly visible discontinuous bands in the field of view; class 2 is assigned to the cases when the number of such bands is not more than 3; class 3 is for the structure with more than 3 bands; class 4 means 3 bands located close to each other uniformly. The image of centerline segregation is a periodic signal and for evaluation of its parameters we propose a spectral analysis using the Fourier transform. In order to see the image of microstructure of the center area at magnification $\times 200$, the average values of the grayscale level are calculated on the secants parallel to the rolling direction $(\mathrm{M}(\mathrm{y}))$. Deviations of these values reflect the presence of dark bands in the image. The spectrum of the $(\mathrm{M}(\mathrm{y}))$ function is calculated using the discrete Fourier transform, established via the expert evaluation method so that the amplitude of harmonics in the frequency range between 0 and $0.05 \mu^{-1}$ describs the centerline segregation degree in accordance with standard charts in optimal way.

References:

[1] A.A. Kazakov and N.H. Luong, Materials Characterization 46 (2001). No. 2-3, p. 155

[2] A.A. Kazakov and D.V. Kiselev, CIS Iron and Steel Review, No. 1-2 (2007), p. 40.

[3] A.A. Kazakov et al., CIS Iron and Steel Review, 2012, p. 4.

[4] A.A. Kazakov et al., Microscopy \& Microanalysis, 17 (2011), p. 1024.

[5] A.A. Kazakov et al., Microscopy \& Microanalysis, 17 (2011), p. 1744. 